\title{
A longitudinal investigation of whether oral health status has an impact on future dietary intake in older adults in Northern Ireland
}

\author{
D. Logan ${ }^{1}$, C.T. McEvoy ${ }^{1}$, G. McKenna ${ }^{1}$, F. Kee ${ }^{1}$, G. Linden ${ }^{1}$ and J.V. Woodside ${ }^{1}$ \\ ${ }^{1}$ Centre of Excellence for Public Health, School of Medicine, Dentistry and Biomedical Sciences, Queens University \\ Belfast, Belfast, BT12 6BA.
}

This abstract was awarded the FSAI prize for best poster presentation.

Evidence linking oral health status and diet quality in older adults is inconsistent and limited by the few prospective studies conducted to date ${ }^{(1-3)}$. This study investigated whether oral health status had an impact on future dietary intake in older Northern Irish (NI) men from the ongoing Prospective Epidemiological Study of Myocardial Infarction (PRIME).

The study involved older men $[n=1096$, mean $=63 \cdot 5(0 \cdot 09)$ years] who underwent a dental examination at baseline (2001-2004). Oral health status was defined in three broad categories: dentate without dentures $(n=552)$, dentate with dentures $(n=465)$ and edentate with dentures $(n=79)$. A food frequency questionnaire administered in 2015 was used to derive intakes of major food groups and diet quality scores were characterised by the Dietary Diversity Score (DDS) (range 0-8) and Mediterranean Diet Score (MDS) (range $0-8$ ). Associations between oral health status, food intakes and diet quality scores were assessed using regression models adjusted for potential confounders (age, socio-economic status, smoking, alcohol, BMI, diabetes and C-reactive protein).

In fully adjusted models, mean fruit intake (portions/day) was significantly higher in men who were dentate without dentures $[1 \cdot 13$ (95\% CI: 1.05, 1.22)] compared to those who were dentate with dentures [1.07(95\% CI: $0.98,1 \cdot 16)]$ and edentate with dentures [0.78 $(95 \%$ CI: $0.60,0.98)](P=0.008 ; \mathrm{P}$ trend $=0.002)$. Similarly, mean daily intake of nuts was significantly higher in men who were dentate without dentures $[0 \cdot 17(95 \% \mathrm{CI}: 0 \cdot 15,0 \cdot 19)]$ compared to those who were dentate with dentures [0.12(95\% CI: $0 \cdot 10,0 \cdot 15)]$ and edentate with dentures [0.10(95\% CI: 0.04, 0.17)] $(\mathrm{P}=0.002 ; \mathrm{P}$ trend $=0.08)$. Other food groups (olive oil/rapeseed oil, vegetables, oily fish, wine, red meat and wholegrains) were not significantly associated with oral health status. Compared to dentate men without dentures, those who were edentate with dentures were $62 \%$ less likely to achieve the UK dietary guideline of 2 portions fruit/day [OR $0 \cdot 38(95 \%$ CI: $0 \cdot 17,0 \cdot 81 ; \mathrm{P}=0.01)$ ]. In fully adjusted models, mean MDS was significantly higher in men who were dentate without dentures $[4.42(0.08)]$ compared to those who were dentate with dentures $[4 \cdot 17(0 \cdot 09)]$ and edentate with dentures $[3 \cdot 86(0 \cdot 25)](P=0 \cdot 03$; $\mathrm{P}$ trend $=0.04)$. Similarly, higher DDS was observed in dentate men without dentures compared to the other dental groups, but the associations were not significant in fully adjusted models.

In older NI men, oral health status was associated with future dietary intake ( $>10$ years later). Specifically, dentures were associated with lower fruit and nut intake, as well as decreased dietary quality. Therefore, interventions to optimise oral health status could help improve diet quality in older men.

1. Gaewkhiew P, Sabbah W \& Bernabé E (2017) Does tooth loss affect dietary intake and nutritional status? A systematic review of longitudinal studies. $J$ Dent 67, 1-8.

2. Kazemi S, Savabi G, Khazaei S et al. (2011) Association between food intake and oral health in elderly: SEPAHAN systematic review no. 8. Dent Res $J 8$ (Suppl 1), S15-20.

3. Toniazzo MP, de Sant'Ana Amorim P, Muniz FWMG et al. (2017) Relationship of nutritional status and oral health in elderly: Systematic review with meta-analysis'. Clin Nutr $1-7$. 